\title{
UDP-glucuronosyltransferases mediate coffee-associated reduction of liver fibrosis in bile duct ligated humanized transgenic UGT1A mice
}

\author{
Steffen Landerer, Sandra Kalthoff, Christian P. Strassburg \\ Department of Internal Medicine I, University Hospital Bonn, Venusberg-Campus 1, Bonn, Germany \\ Contributions: (I) Conception and design: CP Strassburg; (II) Administrative support: CP Strassburg; (III) Provision of study materials or patients: CP \\ Strassburg; (IV) Collection and assembly of data: S Landerer; (V) Data analysis and interpretation: All authors; (VI) Manuscript writing: All authors; \\ (VII) Final approval of manuscript: All authors. \\ Correspondence to: Christian P. Strassburg, MD. Department of Internal Medicine I, University Hospital Bonn, Venusberg-Campus 1, 53127 Bonn, \\ Germany. Email: christian.strassburg@ukbonn.de.
}

Background: Coffee consumption has been shown to reduce the risk of liver fibrosis and is capable of inducing human UDP-glucuronosyltransferase (UGT) $1 A$ genes. UGT1A enzymes act as indirect antioxidants catalyzing the elimination of reactive metabolites, which in turn are potent initiators of profibrotic mechanisms. The aim of this study was to analyze the role of UGT1A genes as effectors of the protective properties of coffee in bile duct ligation (BDL) induced liver fibrosis.

Methods: Fourteen days BDL with and without coffee pre- and co-treatment was performed in btgUGT1A-WT and $b t g U G T 1 A-S N P$ mice. Hepatic UGT1A mRNA expression levels, serum bilirubin and aminotransferase activities were determined. Liver fibrosis was assessed by collagen deposition, computational analysis of Sirius red tissue staining and expression of profibrotic marker genes. Oxidative stress was measured by hepatic peroxidase concentrations and immunofluorescence staining.

Results: UGT1A transcription was differentially activated in the livers of $b \operatorname{tg} U G T 1 A$-WT mice after BDL, in contrast to a reduced or absent induction in the presence of SNPs. Co-treated (coffee + BDL) btgUGT1AWT-mice showed significantly increased UGT1A expression and protein levels and a considerably higher induction compared to water drinking WT mice (BDL), whereas in co-treated btgUGT1A-SNP mice absolute expression levels remained below those observed in $b \operatorname{tg} U G T 1 A$-WT mice. Collagen deposition, oxidative stress and the expression of profibrotic markers inversely correlated with $U G T 1 A$ expression levels in btgUGT1A-WT and SNP mice after BDL and coffee + BDL co-treatment.

Conclusions: Coffee exerts hepatoprotective and antioxidative effects via activation of UGT1A enzymes. Attenuated hepatic fibrosis as a result of coffee-mediated UGT1A induction during cholestasis was detected, while the protective action of coffee was lower in a common low-function UGT1A SNP haplotype present in $10 \%$ of the Caucasian population. This study suggests that coffee consumption might constitute a potential strategy to support the conventional treatment of cholestasis-related liver diseases.

Keywords: Glucuronidation; cholestasis; liver fibrosis; coffee; oxidative stress

Submitted Jan 06, 2020. Accepted for publication Apr 13, 2020.

doi: 10.21037/hbsn-20-9

View this article at: https://dx.doi.org/10.21037/hbsn-20-9 


\section{Introduction}

Coffee is believed to have been first discovered in a region of southwest Ethiopia called Kaffa and has seen as an unprecedented rise to become one of the most widely consumed beverages worldwide $(1,2)$. With about $75 \%$ of the American population consuming coffee, the annual per-capita consumption in the United States 2015 exceeded $5 \mathrm{~kg}$ of green coffee (3). Besides its sought-after taste and stimulating effect, coffee has been associated with hepatoprotective properties. An increasing number of epidemiological studies have reported that coffee consumption is inversely associated with fibrosis progression, hepatic cirrhosis and hepatocellular carcinoma (HCC) (4-6).

Previous data from our own laboratory identified coffee as an efficient activator of UDP-glucuronosyltransferase (UGT) 1A expression (7). UGT1A enzymes eliminate a wide range of endo- and xenobiotic compounds, including many reactive metabolites, thereby acting as indirect antioxidants and contribute to cytoprotection (8). The UGT1A-mediated conjugation of substrates with glucuronic acid leads to the formation of water soluble, biologically inactive glucuronides and facilitates subsequent excretion via bile and urine (9-11). Transcription of UGT1A genes is known to be regulated by tissue-specific and ligandactivated transcription factors including bile acid activated farnesoid X-receptor (FXR), oxidative stress sensor Nrf2 (nuclear factor erythroid 2-related factor 2) and xenobiotic inducible aryl-hydrocarbon receptor (AhR) (12-14). UGT1A expression and activity is further influenced by the presence of single nucleotide polymorphisms (SNPs). With more than 100 identified UGT1A SNPs, which exhibit frequencies of up to $40 \%$ in the Caucasian population, a huge variety of genetic variants affecting protein function and transcriptional activation has been described so far $(15,16)$. Polymorphisms leading to reduced UGT1A function have been identified as risk factors for cancer (17) and are associated with more severe fibrosis progression in patients with hepatitis $C(18,19)$.

Apart from HCV infection, alcohol consumption and non-alcoholic steatohepatitis, cholestasis-related liver injury represents one of the major causes of hepatic fibrosis and cirrhosis in industrialized countries $(20,21)$. Cholestasis induced fibrosis is characterized by a massive accumulation of extracellular matrix (ECM) proteins, an increase of inflammatory and other cytokines, as well as elevated oxidative stress levels (22-24). In a recent study, we were able to show that the coffee-mediated protective effects towards benzo(a)pyrene-induced oxidative stress are dependent on the presence of UGT1A enzymes (25). Aim of the study was therefore to show that the induction of UGT1As via coffee ameliorates the effects of chronic cholestatic liver injury. To this end an extreme model of advanced hepatic cholestasis was selected. Humanized transgenic (btg) UGT1A mice underwent surgical ligation of the common bile duct (bile duct ligation, BDL) leading to the subsequent retention of toxic bile acids and thus liver fibrosis within 14 days.

\section{Methods}

\section{Surgery and treatment of humanized transgenic UGT1A mice}

In this study two previously reported and characterized $b t g$ mouse lines were used. The $b t g U G T 1 A-W T$ line, containing the wild type (WT) UGT1A gene locus, and a line containing a haplotype of 10 common UGT1A SNPs (UGT1A1*28, UGT1A3 -66T>C, UGT1A3 W11R, UGT1A3 V47A, UGT1A6*2a (S7A/T181A/R184S), UGT1A7*3 (N129K/R131K/W208R/-57T>G)) (26). This SNP mouse model was created to simulate a frequent variant haplotype observed in $10 \%$ of the Caucasian population. Studies have shown that many of these SNPs are simultaneously present in Gilbert syndrome individuals and influence the glucuronidation by modifying the transcriptional activation (27) and/or by expressing proteins with altered enzymatic activity $(28,29)$. A detailed overview of the SNPs included in the $b t g U G T 1 A$-SNP mouse line and the respective minor allele frequency (MAF) is shown in Table 1. For animal experiments 8-12-week-old female btgUGT1A-WT and SNP mice were used. Mice of each genotype were divided into four groups of four to six animals and either underwent 14 days BDL or 14 days sham operation. In addition to BDL or sham operation, btgUGT1A-WT and SNP mice received pre- and cotreatment with coffee as their drinking water 14 days before and after surgical treatment.

BDL was performed using a standard technique as described elsewhere (35). Mice were anesthetized by inhalation of $4 \mathrm{vol} \%$ isoflurane in $100 \%$ oxygen at a flow rate of $4 \mathrm{~L} / \mathrm{min}$ and $1.5-3 \mathrm{vol} \%$ isoflurane at a flow rate of $1 \mathrm{~L} / \mathrm{min}$ was set to maintain anaesthesia. After midline laparotomy $(1.5 \mathrm{~cm})$ the common bile duct was exposed and ligated twice with a surgical knot. In sham operated mice, 


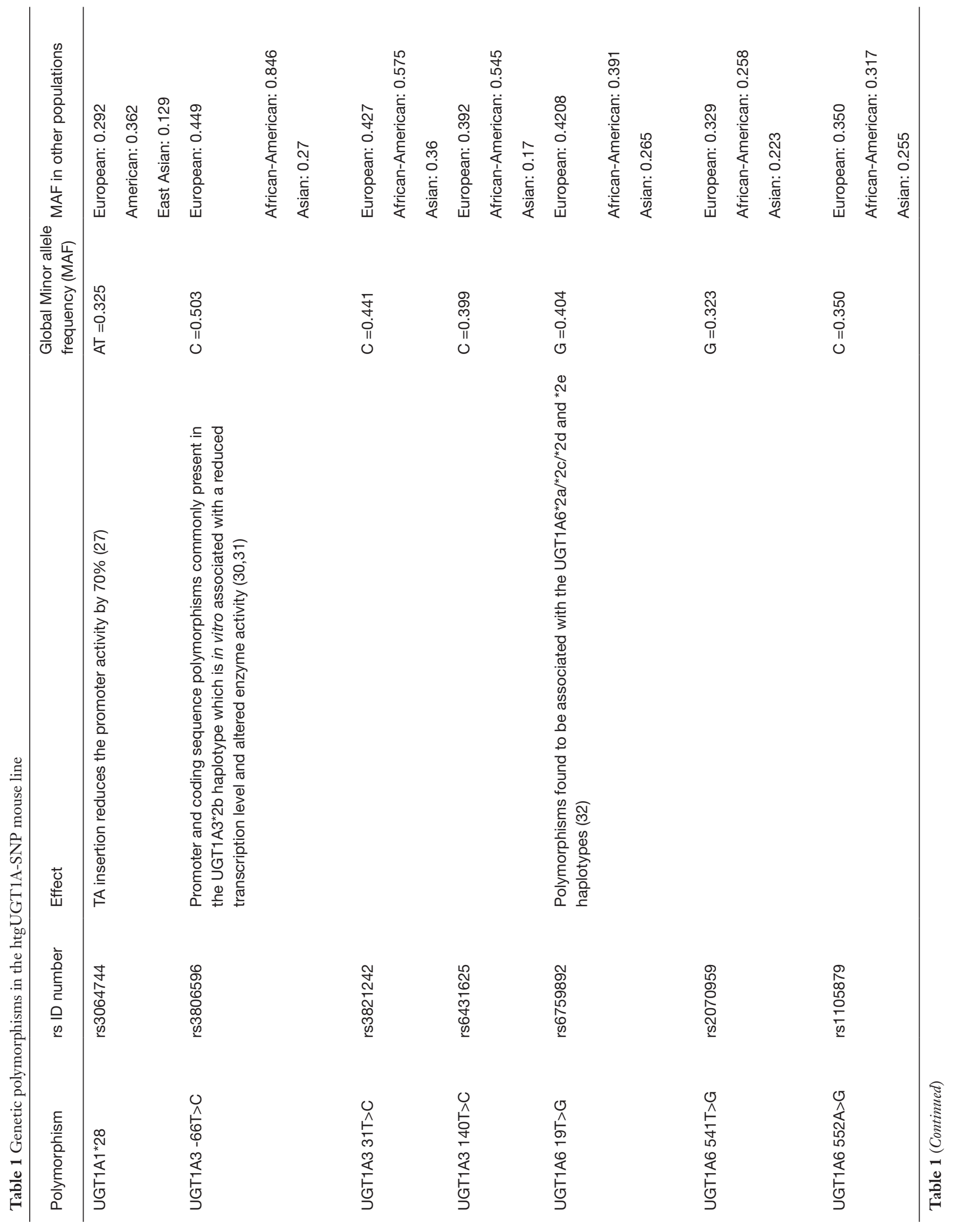




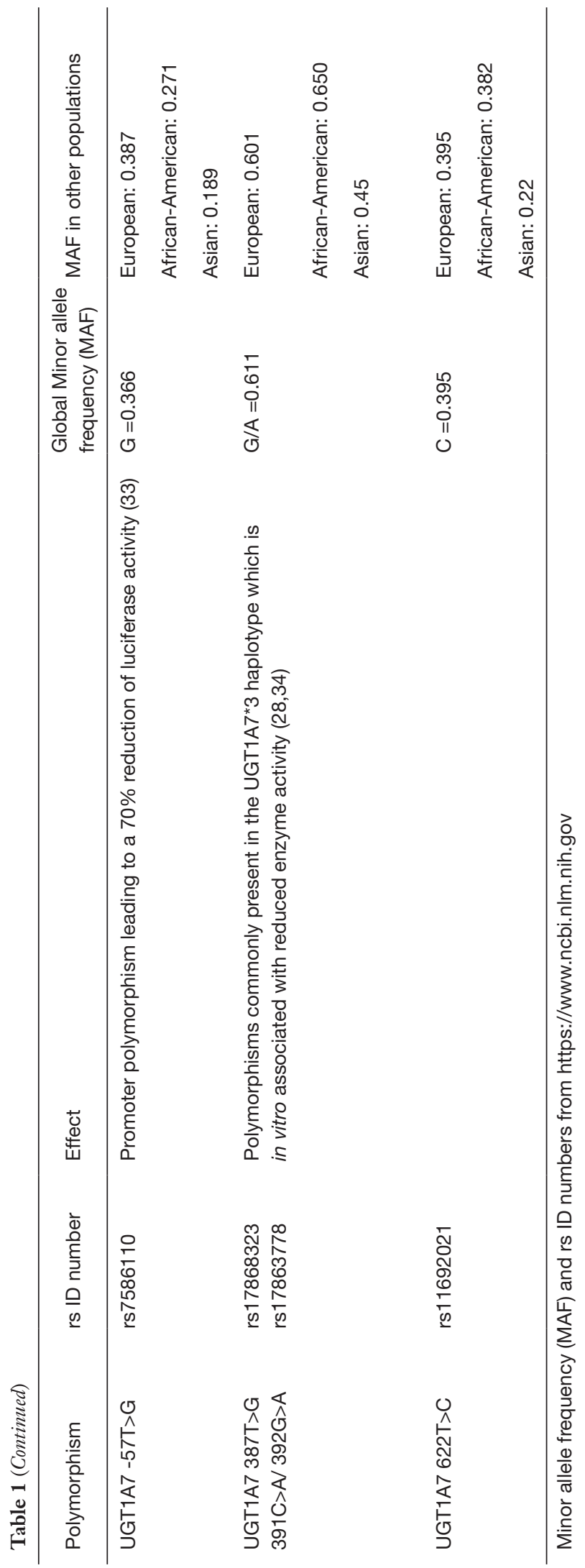

the bile duct has not been ligated. 14 days after surgery, blood and organs were collected, shock-frozen in liquid nitrogen and stored at $-80^{\circ} \mathrm{C}$ until use.

Animals were housed in the Central Animal Facility of the University Hospital Bonn and were kept at $22{ }^{\circ} \mathrm{C}$ with a $12 \mathrm{~h}$ day/night cycle with ad libitum access to chow. All experiments were performed in accordance to the "German Animal-Protection Law" and the relevant guidelines of the Local Institutional Animal Care unit of our university (Haus für experimentelle Therapie, Bonn, Germany) and authorized by the relevant North Rhine-Westphalian stateagency for Nature, Environment and Consumer Protection (LANUV, Germany) under the file reference LANUV 8402.04.2016.A483.

\section{Standardized preparation of coffee}

A stock solution was prepared to represent the preparation mode of commonly used filter coffee. For this purpose $500 \mathrm{~mL}$ water (Aqua Irrigation Solution, DeltaSelect, Dreieich, Germany) were boiled in a beaker and cooled for 10 seconds. Ten grams of ground coffee powder (Jacobs Krönung, Kraft Foods, Bremen, Germany) were added and incubated for one min, followed by filtration through a paper coffee filter (Melitta, Minden, Germany).

\section{Measurement of alanine aminotransferase (ALT), aspartate aminotransferase (AST) and bilirubin}

Total serum bilirubin levels, ALT and AST were measured using a Fuji DRI-CHEM NX500i (Fujifilm Cooperation, Tokyo, Japan) serum analyser. After collecting blood, it was centrifuged at 13,000 $\mathrm{g}$ for $10 \mathrm{~min}$ and supernatant was subsequently stored at $-20^{\circ} \mathrm{C}$ until measurement.

\section{Gene expression analysis}

Total RNA was isolated from shock-frozen liver tissue of sacrificed transgenic mice and homogenized in TRIzol (Invitrogen, Karlsruhe, Germany). For further analyses equal amounts from at least four animals per group were pooled. $5 \mu \mathrm{g}$ of RNA was incubated with DNase I (Invitrogen) at room temperature for $15 \mathrm{~min}$ and subsequently inactivated at $65^{\circ} \mathrm{C}$ for $10 \mathrm{~min}$. DNase I treated RNA was then used for cDNA synthesis in an oligo(dT)-primed Superscript III reverse transcriptase reaction according to the manufacturer's instructions (Fischer Scientific, Schwerte, Germany). For quantification 
of gene expression, cDNA concentrations were determined by qPCR relative to mouse beta-actin. Using gene specific primers and probes $\mathrm{qPCR}$ reactions were performed in CFX96 real-time PCR detection system (Bio-Rad) with qPCR MasterMix (Eurogentec). All reactions were performed in triplicates and have been repeated three times. Bio-Rad CFX Manager 3.0 software was used to calculate the relative expression.

\section{Histological analysis}

Liver fibrosis was assessed by computational analysis of Sirius red stained areas. For the detection of collagen fibres, paraffin-embedded sections were trimmed to $2.0 \mu \mathrm{m}$ slices and stained in Sirius red solution (saturated picric acid containing $0.1 \%$ DirectRed 80 ). The Sirius red positive area was quantified using ImageJ software (U.S. National Institutes of Health; http://rsb.info.nih.gov/ij/) and shown as percentage of the total section area. Images were analysed from four randomly picked pictures (magnification 100x) of each animal and averaged. The quantitative analysis of fluorescence intensity obtained from immunofluorescence images of UGT1A protein (magnification 200x) was also calculated with the ImageJ program and shown as relative fluorescence units (RFU).

For analyzation of UGT1A protein levels and for the determination of lipid peroxidation secondary immunofluorescence staining was performed. As described elsewhere (36) deparaffinization, rehydration and antigen retrieval of paraffin embedded tissue slides was accomplished by incubation of liver specimens in decreasing alcohol concentrations followed by $20 \mathrm{~min}$ heating in sodium citrate buffer $\mathrm{pH} 6.0$ at $95-100{ }^{\circ} \mathrm{C}$ and then washed three times before being blocked with blocking buffer (1× $\mathrm{PBS} / 5 \%$ goat serum) for $1 \mathrm{~h}$. Overnight incubation with respective primary antibodies [anti 4 hydroxynonenal (4HNE), Abcam ab46545, 1:50 and anti UGT1A, Santa Cruz sc-271268, 1:50] was carried out in TBS-T containing $5 \%$ goat serum. Appropriate secondary antibodies (Alexa Fluor $^{\circledR} 488$ Abcam ab150077 and ab150113, dilution 1:200 each) were added to tissue sample area for $1 \mathrm{~h}$. A mounting medium with DAPI (Abcam) was applied according to manufacturer's instructions. The specimens were visualized under a microscope (Axio Scope.A1, Zeiss) at the same day.

\section{Peroxidase assay}

For the colorimetric determination of total hepatic peroxidase concentrations, $100 \mathrm{mg}$ liver tissue was homogenized and evaluated with the use of OxiSelect ${ }^{\text {TM }}$ Hydrogen Peroxidase Assay Kit (Cell Biolabs, Inc.) according to manufacturer's protocol. Samples were analysed using Multiskan Go Reader (ThermoScientific).

\section{Statistical analysis}

Data are expressed as mean \pm standard deviation (SD) determined by one-way analysis of variance followed by Student's $t$-test to determine significance. A pool of four to six mice in each group was analysed, $\mathrm{P}$ values below 0.05 were considered as statistically significant.

\section{Results}

\section{Coffee reduces liver fibrosis in BDL-treated btgUGT1A- WT mice}

In order to examine coffee-mediated effects during obstructive cholestasis, BDL btgUGT1A-WT mice were analysed for hepatic collagen deposition, total serum bilirubin levels and aminotransferase activities. Sirius red staining in btgUGT1A-WT mice confirmed fibrosis development 14 days after BDL, as well as a clearly visible reduction of red stained collagen fibres in coffee co-treated BDL mice (Figure 1A). As expected, bile duct ligated btgUGT1A-WT mice showed significantly elevated total serum bilirubin levels $(23.02 \mathrm{mg} / \mathrm{dL})$ compared to sham operated mice $(1.2 \mathrm{mg} / \mathrm{dL})$. Interestingly, administration of coffee significantly reduced $(31 \%)$ total serum bilirubin levels compared to water drinking BDL mice. Furthermore, measurement of serum aminotransferase levels revealed a significant elevation of AST (23.6-fold) and ALT (50.1fold) levels after BDL, whereas coffee treatment led to a considerable reduction of AST (49\%) and ALT (54\%) levels in comparison to the water drinking BDL group (Figure 1B-1D). Even though serum aminotransferase activities still were significantly elevated after coffee + BDL co-treatment (AST: 11.5-fold and ALT: 32.8-fold), these data demonstrate that coffee exerts a protective effect during obstructive cholestasis-induced liver damage.

\section{Hepatic UGT1A expression during cholestatic liver fibrosis is differentially regulated in coffee exposed btgUGT1A- WT mice}

Coffee administration in sham operated btgUGT1A- 


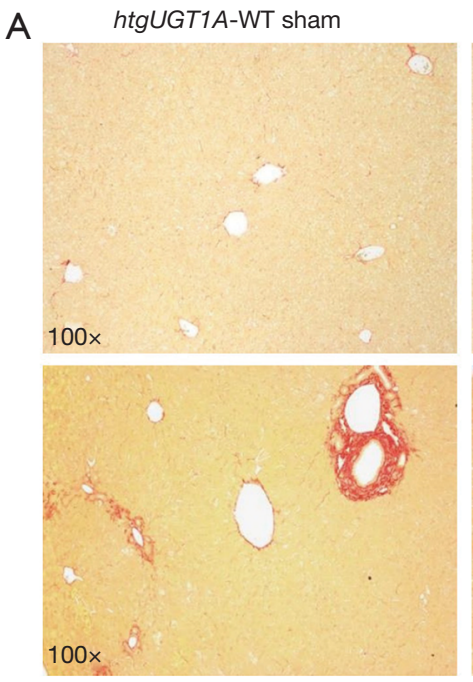

htgUGT1A-WT 14 days BDL

B

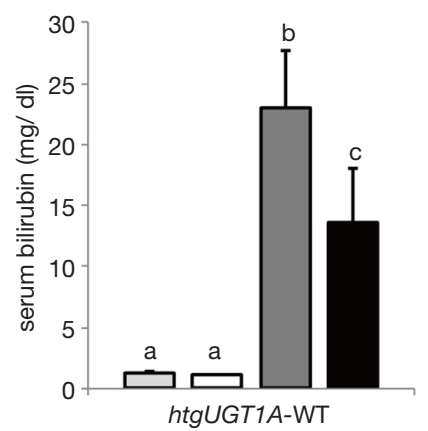

htgUGT1A-WT coffee \& sham
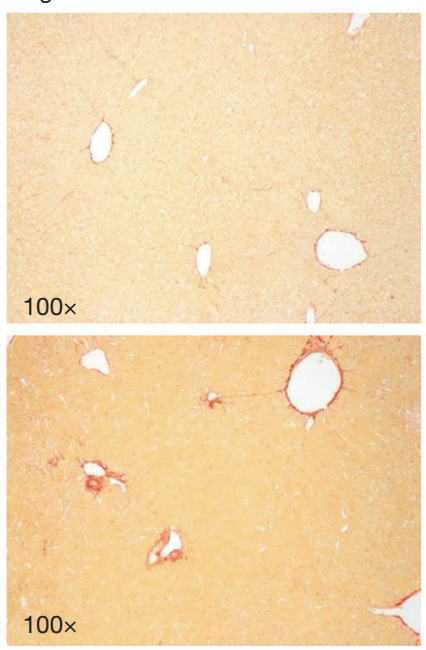

htgUGT1A-WT coffee \& 14 days BDL

$\mathrm{C}$

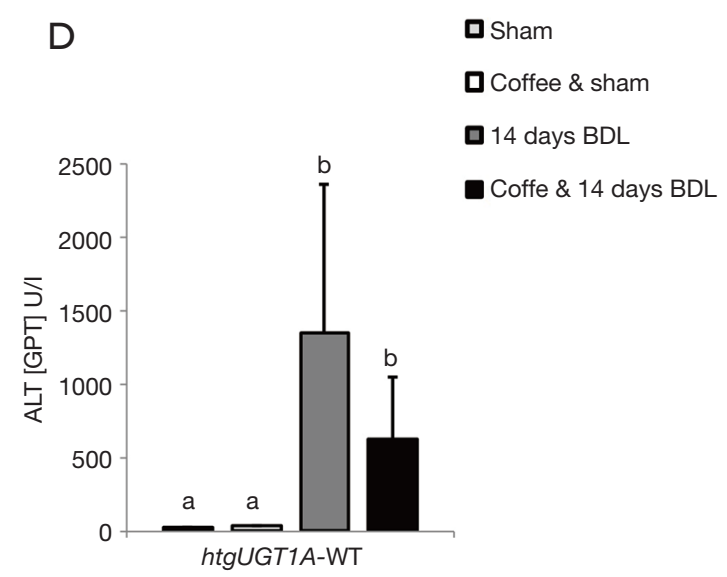

Figure 1 Histological evaluation and biochemical serum analyses of btgUGT1A-WT mice. Representative hepatic sections of histological Sirius red staining in $h \operatorname{tg} U G T 1 A$-WT mice (A, magnification 100x); determination of total serum bilirubin levels (B), aspartate aminotransferase (C) and alanine aminotransferase (D) activities in serum of sham operated (sham) and bile duct ligated (BDL) btgUGT1AWT mice with and without coffee pre- and co-treatment. Graphs are expressed as means \pm SD using 4 mice per sham group and 6 mice in each BDL group. Samples were analyzed with Student's $t$-test. Means with different letters indicate significant differences at $\mathrm{P}<0.05$, and columns sharing the same letter are not significantly different. AST, aspartate aminotransferase; ALT, alanine aminotransferase.

WT mice led to a significant hepatic mRNA induction of all investigated UGT1A isoforms (except for UGT1A7) compared to water drinking sham operated animals (Figure 2). After biliary obstruction, an upregulation of UGT1A1-, UGT1A6-, UGT1A7 and UGT1A9 mRNA expression compared to the sham operated group was observed. UGT1A gene expression in the livers of btgUGT1A-WT mice was significantly increased after coffee + BDL co-treatment and showed considerable higher induction compared to water drinking BDL mice for the isoforms UGT1A1 (7.1-fold), UGT1A3 (2.3-fold),
UGT1A4 (1.8-fold), UGT1A6 (4.3-fold), UGT1A7 (5.9fold) and UGT1A9 (3.2-fold). These data indicate that BDL-induced liver fibrosis differentially activates hepatic glucuronidation. Coffee co-treatment further points to a considerable responsiveness of transcriptional $U G T 1 \mathrm{~A}$ activation, resulting in a synergistic inductive effect in btgUGT1A-WT mice. Immunofluorescent detection of UGT1A proteins confirmed the results measured at the mRNA level demonstrating the considerable induction of UGT1A protein after coffee administration and coffee $+\mathrm{BDL}$ co-treatment in comparison to equivalent treated 

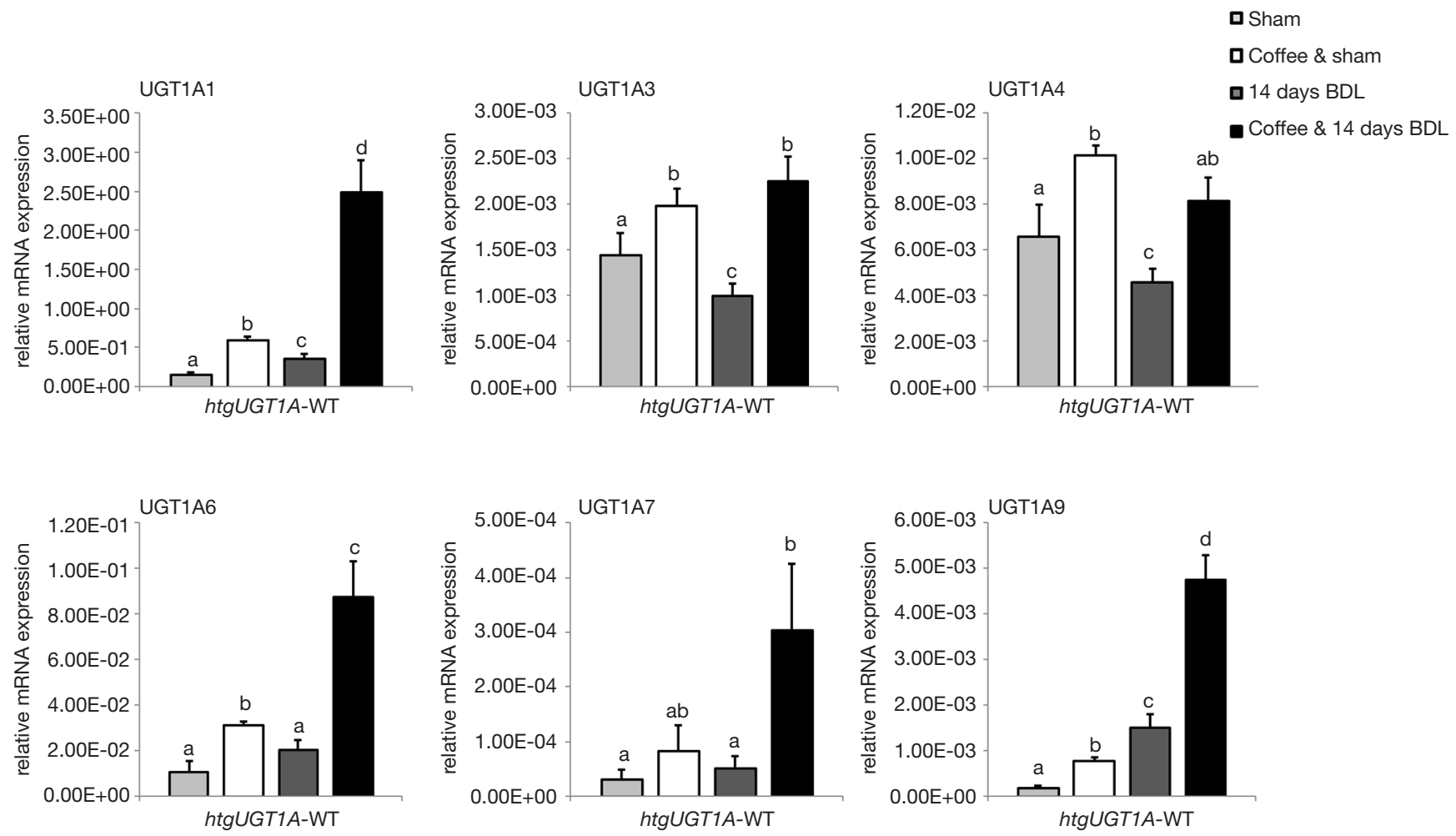

Figure 2 Hepatic UGT1A mRNA expression in btgUGT1A-WT mice after sham operation (sham) or 14 days bile duct ligation (BDL) with and without coffee pre- and co-treatment. Graphs are expressed as means \pm SD using 4 mice per sham group and 6 mice in each BDL group. Samples were analyzed with Student's $t$-test. Means with different letters indicate significant differences at $\mathrm{P}<0.05$, and columns sharing the same letter are not significantly different.

btgUGT1A-WT mice drinking water (Figure $3 A$ ). The quantitative analysis of fluorescence intensity obtained from the depicted UGT1A immunofluorescent images further substantiated these results. The calculated mean of intensity also basically corresponded to the determined UGT1A mRNA levels revealing the lowest relative fluorescence intensity after sham operation and the highest intensity in coffee + BDL co-treated btgUGT1A-WT mice (Figure 3B).

\section{Coffee reduces collagen deposition during cholestatic liver injury in btgUGT1A-WT and SNP mice}

To further elucidate the role of UGT1A expression in coffee-mediated fibrosis protection during BDL treatment, the $b \operatorname{tg} U G T 1 A$-SNP mouse line known for its reduced basal UGT1A expression and inducibility was used (26). Computational quantification of Sirius red stained tissues was performed and both btgUGT1A mouse lines were compared for their ECM content (Figure 4A). As expected, the direct comparison between BDL btgUGT1A-WT and SNP mice revealed significantly higher ECM deposition in mice carrying the low-activity SNP variant (WT 3.41\% and SNP 4.94\%). Furthermore, coffee-mediated reduction of fibrillar collagen was more pronounced in btgUGT1AWT mice, whereas co-treated SNP mice also exhibited a reduced but still significantly higher content of positively stained areas and consequently showed a more severe fibrosis development evidenced by a higher total ECM deposition (WT 2.63\% and SNP 4.08\%). Expression levels of collagen type 1 alpha 1 (Col1a1) further supported the results obtained in computational Sirius red quantification. Although considerable upregulation of Col1a1 expression was detected after BDL in both mouse lines (WT 12.4fold and SNP 22.2-fold), btgUGT1A-SNP mice showed significantly higher mRNA expression levels (Figure 4B). After simultaneous treatment with coffee and BDL, a downregulation of Col1a1 expression levels in both mouse lines was detected, even though mice containing multiple 

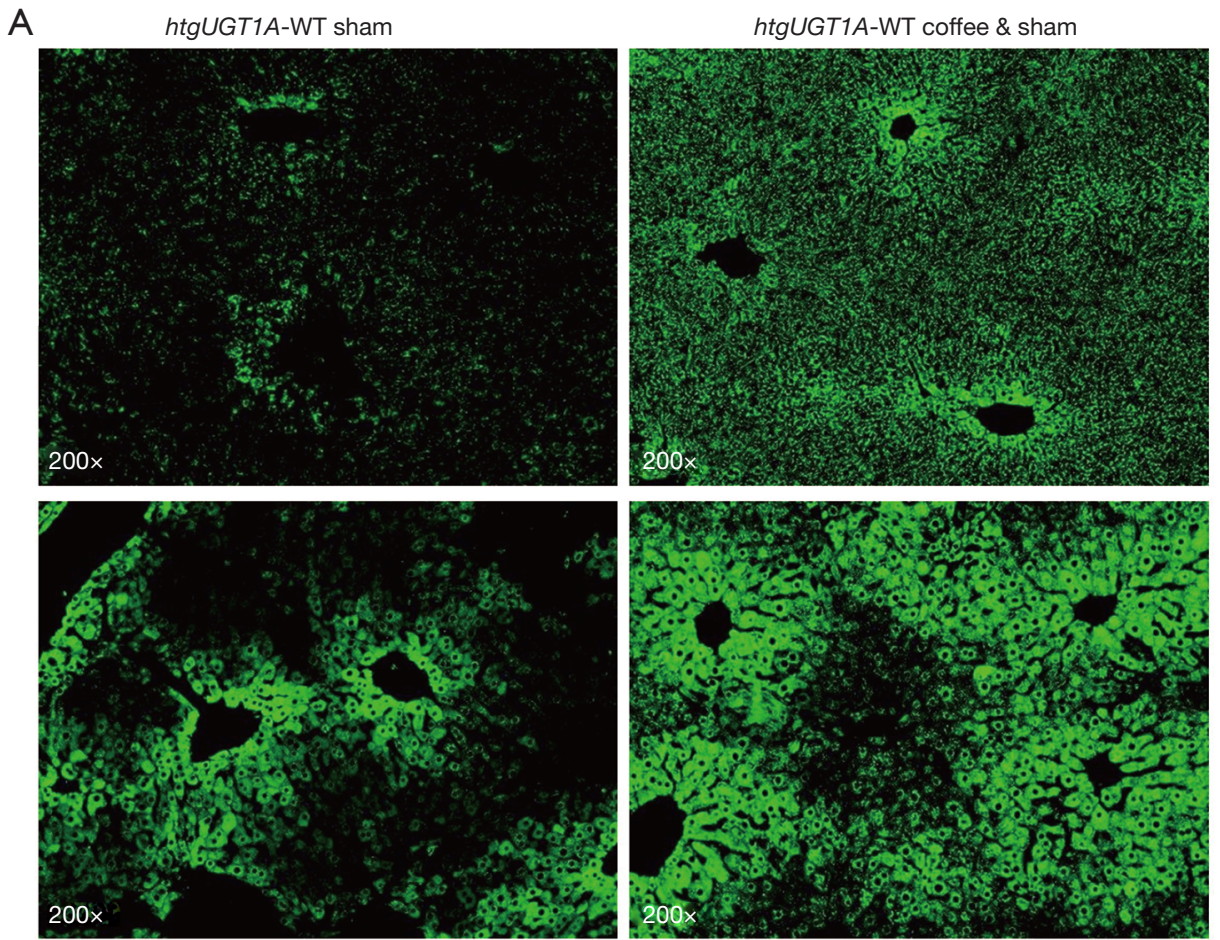

htgUGT1A-WT 14 days BDL

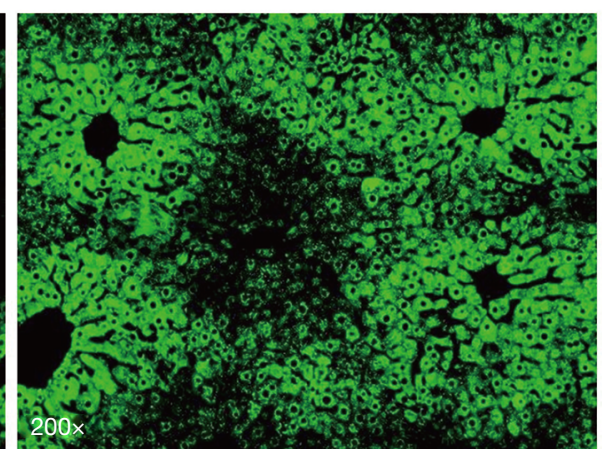

htgUGT1A-WT coffee \& 14 days BDL

B

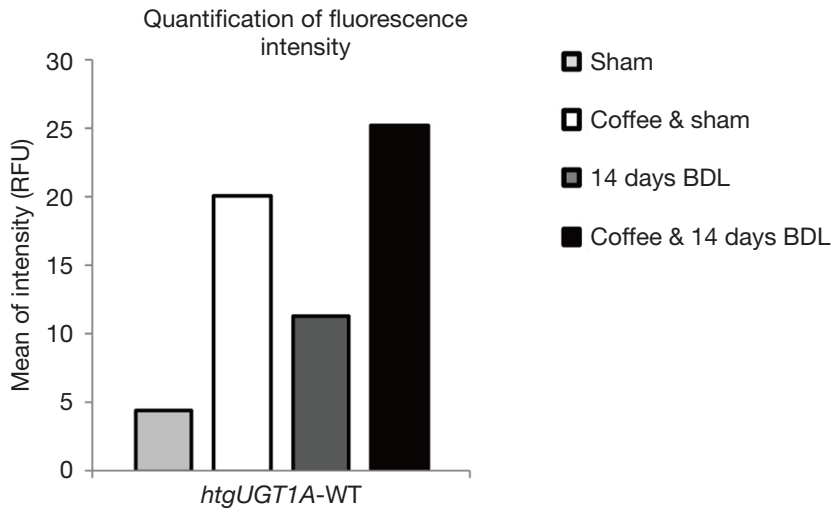

Figure 3 Detection and computational quantification of UGT1A protein in liver tissue of $b t g U G T 1 A$-WT mice. Representative images of UGT1A immunofluorescence staining of btgUGT1A-WT mice after sham operation (sham) or 14 days bile duct ligation (BDL) with and without coffee pre- and co-treatment (A, magnification 200x). Quantitative assessment of fluorescence intensity shown as mean of intensity in relative fluorescence units of the depicted image calculated with Image (B). RFU, relative fluorescence units.

genetic SNP variants still had higher expression levels (1.5fold) compared to their equally treated WT counterparts. These findings were in line with the results observed in Sirius red staining (Figures $1 A, 4 C$ ). In water and coffee drinking BDL btgUGT1A-SNP mice, Sirius red staining revealed advanced hepatic fibrosis, although a diminished proportion of red stained collagen fibres after coffee cotreatment could be observed.

\section{Differential oxidative stress levels after BDL treatment in btgUGT1A-WT and SNP mice}

Oxidative stress results from an imbalance in the production of reactive oxygen species (ROS) and the ability of the organism to scavenge them $(37,38)$. UGT1As act as indirect antioxidants because of their ability to eliminate reactive metabolites capable of inflicting tissue injury (8). Since oxidative stress is a well-known initiator of fibrogenesis, 
A

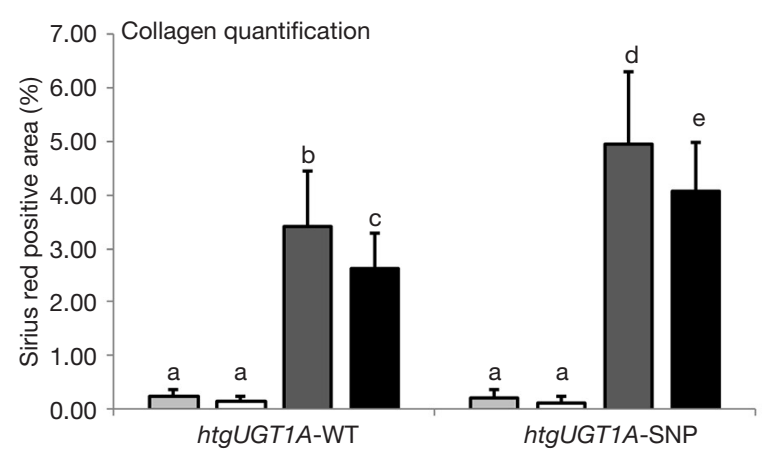

C

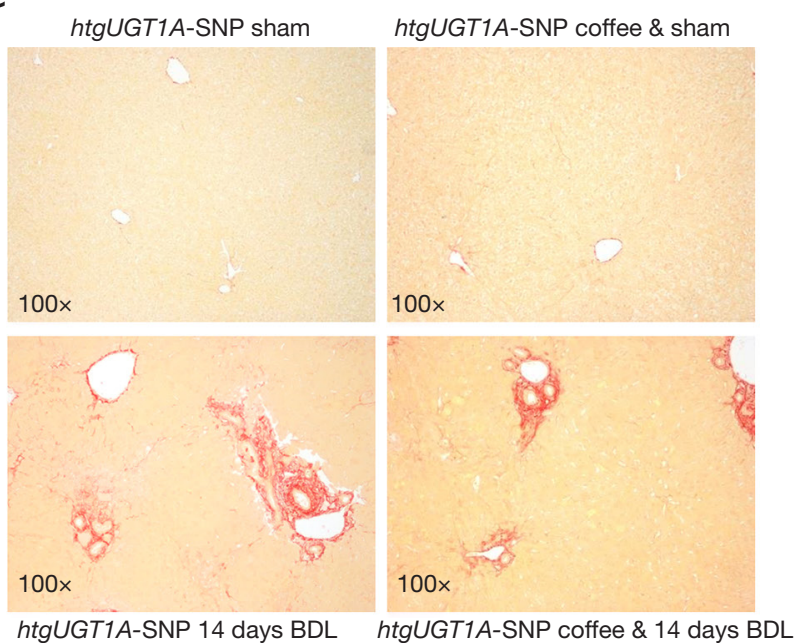

B

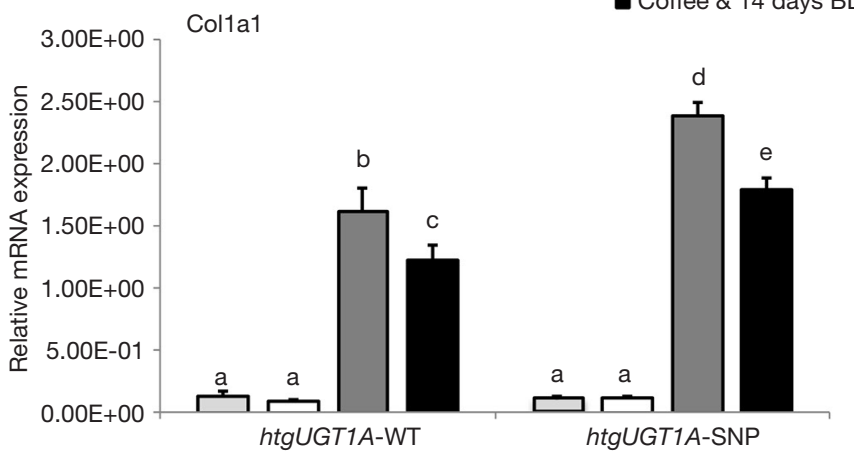

口Sham

口 Coffee \& sham

口 14 days BDL

Coffee \& 14 days BDL

htgUGT1A-SNP

Figure 4 Deposition and expression of hepatic collagens in $b t g U G T 1 A$-WT and SNP mice. Quantification of the Sirius red positive areas of $b t g U G T 1 A$-WT and $b t g U G T 1 A$-SNP mice calculated with ImageJ (A). TaqMan PCR measured mRNA expression levels of Col1a1 after sham operation (sham) or 14 days bile duct ligation (BDL) with and without coffee pre- and co-treatment (B). Representative sections of Sirius red stained areas of $b \operatorname{tg} U G T 1 A$-SNP mice (C, magnification 100x). Graphs are expressed as means \pm SD using 4 mice per sham group and 6 mice in each BDL group. Samples were analyzed with Student's $t$-test. Means with different letters indicate significant differences at $\mathrm{P}<0.05$, and columns sharing the same letter are not significantly different. Col1a1, collagen type 1 alpha 1.

detection of 4-HNE was used as marker for lipid peroxidation and oxidative injury in liver tissue $(39,40)$. As shown in Figure $5 A$, fluorescence intensity of 4-HNE was higher in BDL-treated btgUGT1A-SNP mice compared to mice carrying the human wild type $U G T 1 A$ gene locus. Interestingly, coffee co-treatment nearly abolished the fluorescence signal of 4-HNE detection in btgUGT1AWT mice, whereas in the presence of the UGT1A SNP variant merely a moderate reduction of lipid peroxidation compared to the water drinking BDL group was detected. These results indicate a coffee-mediated increase of the antioxidative capacity, which is more pronounced in mice carrying the $U G T 1 A$ wild type gene locus as indicated by lower lipid peroxidation-caused oxidative injury and confirm a role of UGT1A activity in cellular protection.

In addition, total hepatic peroxidase concentrations, which includes glutathione peroxidase as well-established indicator for oxidative stress (41) was investigated in btgUGT1A-WT and SNP mice (Figure 5B). Following BDL, peroxidase concentrations significantly decreased in btgUGT1A-WT mice (39.2\%), whereas coffee pre- and co-treatment led to significantly higher hepatic peroxidase 

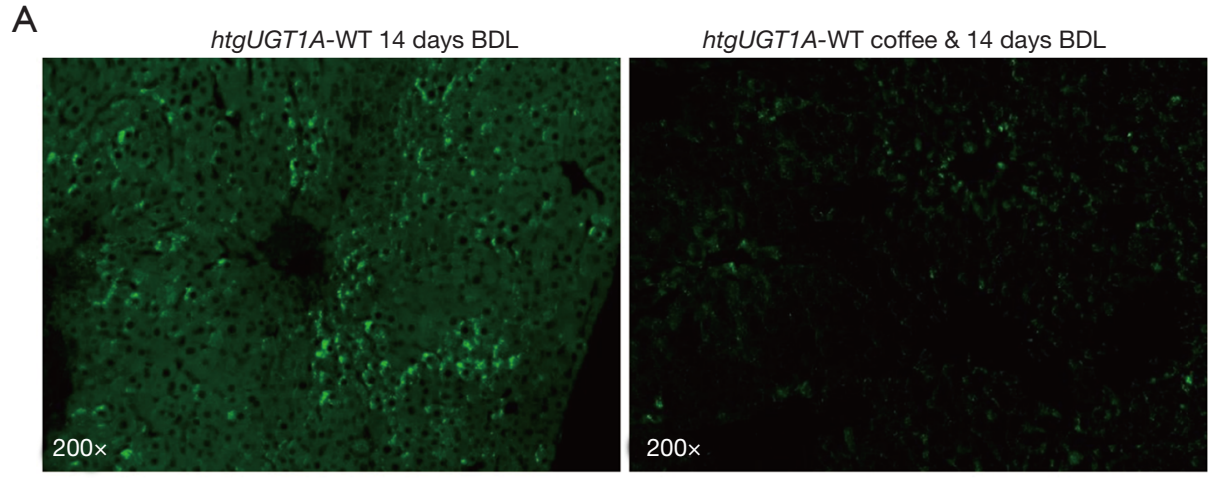

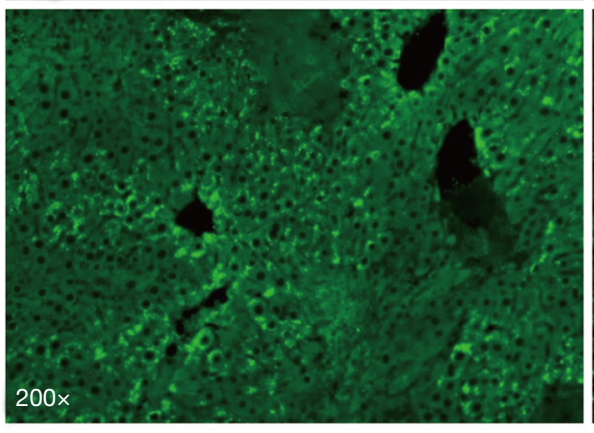

htgUGT1A-SNP 14 days BDL

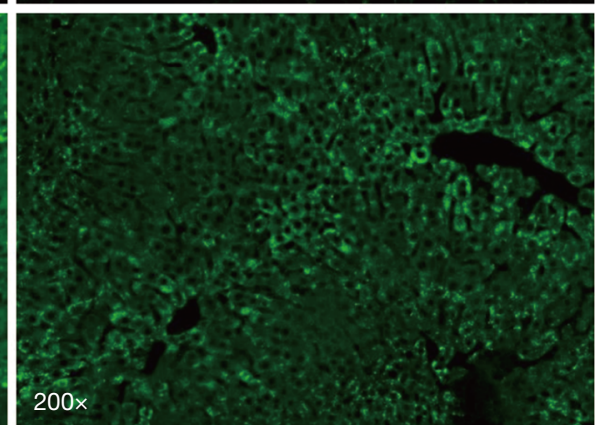

htgUGT1A-SNP coffee \& 14 days BDL

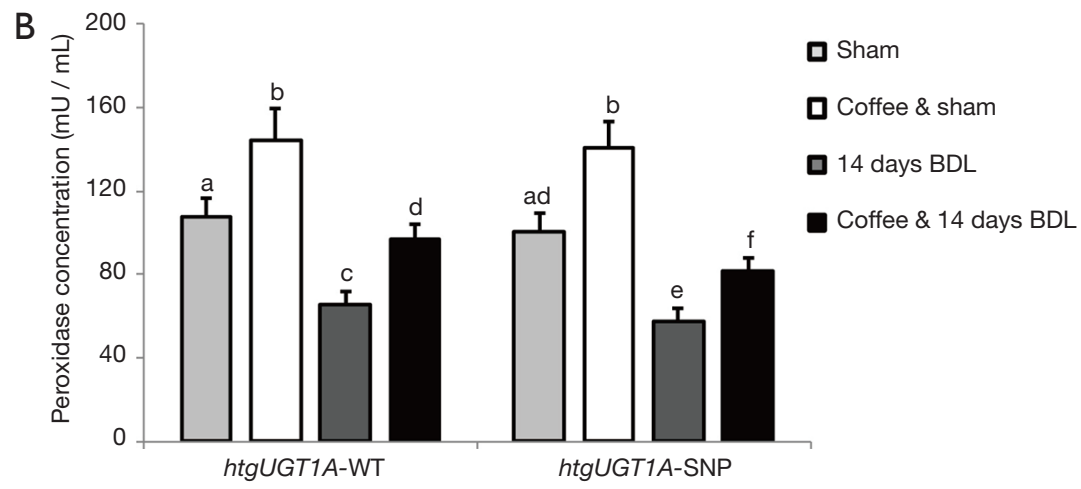

Figure 5 Oxidative liver injury and hepatic oxidative stress levels in btgUGT1A-WT and SNP mice. Representative pictures of lipid peroxidation detection by immunofluorescence staining with 4-HNE antibody (A, magnification 200x), and comparison of total hepatic peroxidase concentrations (B) in btgUGT1A-WT and SNP mice after sham operation (sham) or 14 days bile duct ligation (BDL) with and without coffee pre- and co-treatment. Graphs are expressed as means \pm SD using 4 mice per sham group and 6 mice in each BDL group. Samples were analyzed with Student's $t$-test. Means with different letters indicate significant differences at $\mathrm{P}<0.05$, and columns sharing the same letter are not significantly different. 4-HNE, 4 hydroxynonenal.

concentrations (1.47-fold) compared to water drinking BDL mice. However, peroxidase levels of BDL and coffee co-treated btgUGT1A-WT mice $(65.5$ and $96.6 \mathrm{mU} / \mathrm{mL}$ ) were significantly higher as those observed in the presence of $U G T 1 A$ SNPs $(57.8$ and $81.9 \mathrm{mU} / \mathrm{mL})$. Although coffee co-treatment attenuated oxidative stress in both mouse lines, differences in 4-HNE immunofluorescence detection and total hepatic peroxidase concentrations indicate an essential role of UGT1A function for the coffee-mediated antioxidative effects. As a consequence, an altered modification of the metabolic antioxidative balance in $b \operatorname{tg} U G T 1 A-\mathrm{SNP}$ mice may result in enhanced fibrosis 

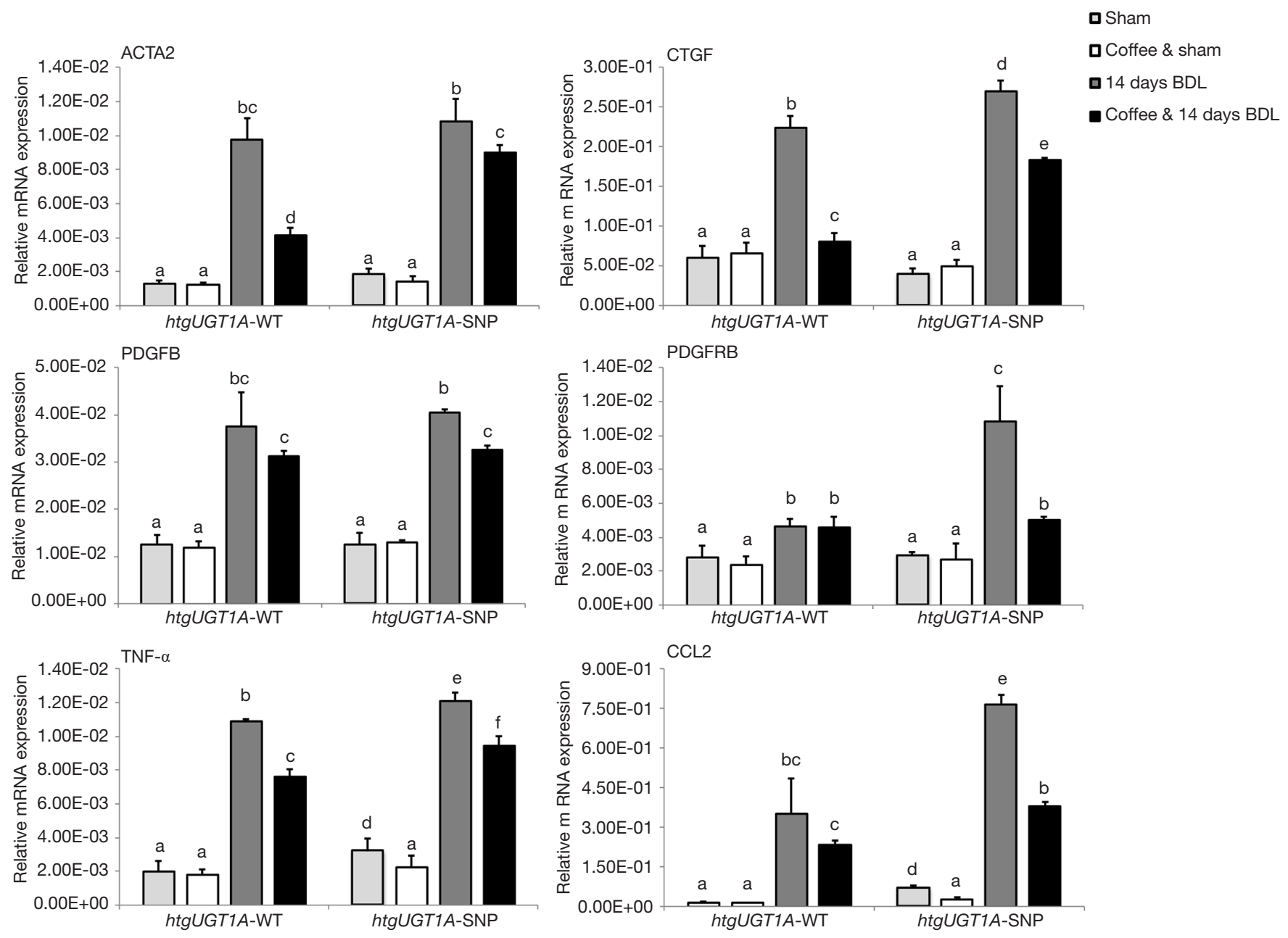

Figure 6 Hepatic expression of profibrotic marker genes in btgUGT1A-WT and SNP mice after sham operation (sham) or 14 days bile duct ligation (BDL) with and without coffee pre- and co-treatment. Graphs are expressed as means \pm SD using 4 mice per sham group and 6 mice in each BDL group. Samples were analyzed with Student's $t$-test. Means with different letters indicate significant differences at $\mathrm{P}<0.05$, and columns sharing the same letter are not significantly different. ACTA2, alpha smooth muscle actin ( $\alpha$-SMA); CTGF, connective tissue growth factor; PDGFRB, beta-type platelet-derived growth factor receptor; PDGFB, platelet-derived growth factor subunit B; TNF- $\alpha$, tumor necrosis factor alpha; CCL2, C-C chemokine ligand 2.

initiation during cholestasis.

\section{Expression of marker genes for fibrosis in BDL and coffee co-treated BDL btgUGT1A-WT and SNP mice}

The development of fibrosis is associated with an increased expression of various cytokines and chemokines, which serve as reliable biomarkers for the fibrogenic activity or hepatic inflammation. In comparison to sham operated mice, 14 days BDL led to a significant transcriptional activation of the profibrotic marker genes alpha smooth muscle actin ( $\alpha$-SMA, ACTA2), connective tissue growth factor (CTGF), beta-type platelet-derived growth factor receptor (PDGFRB) and platelet-derived growth factor subunit B (PDGFB) in the livers of $b t g U G T 1 A$-WT and SNP mice (Figure 6). Furthermore, absolute expression levels of the proinflammatory markers tumour necrosis factor alpha (TNF- $\alpha$ ) and C-C chemokine ligand 2 (CCL2) were also significantly upregulated after 14 days BDL. Comparing the transcriptional activation of profibrotic marker genes in water drinking BDL operated $h t g U G T 1 A-W T$ and SNP mice, significantly increased absolute expression levels for 


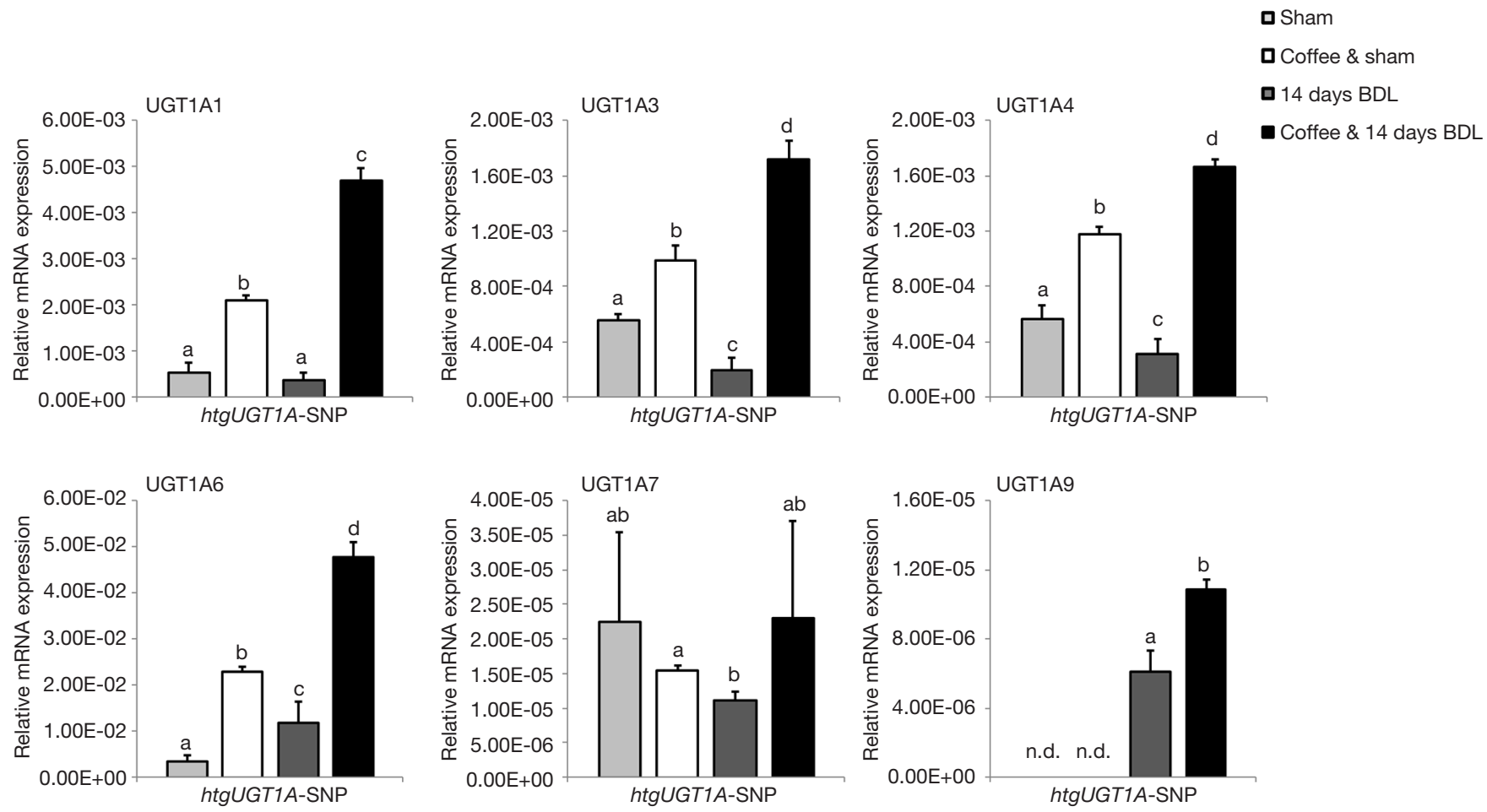

Figure 7 Hepatic UGT1A mRNA expression in $b \operatorname{tg} U G T 1 A$-SNP mice after sham operation (sham) or 14 days bile duct ligation (BDL) with and without coffee pre- and co-treatment. Graphs are expressed as means \pm SD using 4 mice per sham group and 6 mice in each BDL group. Samples were analyzed with Student's $t$-test. Means with different letters indicate significant differences at $\mathrm{P}<0.05$, and columns sharing the same letter are not significantly different. n.d., not detectable.

CTGF (1.2-fold), PDGFRB (2.3-fold), TNF- $\alpha$ (1.1-fold) and CCL2 (2.2-fold) in mice carrying the low-function UGT1A SNP haplotype were detected.

The antifibrotic potential of coffee in a wide-ranging spectrum of chronic liver diseases has been described in numerous studies $(42,43)$. In this respect an inverse relationship between coffee consumption and fibrosis progression has also been shown in recently published data $(44,45)$. In line with these data, coffee + BDL co-treatment reduced absolute expression levels of all depicted profibrotic marker genes (except for PDGFRB) in btgUGT1A-WT mice compared to the water drinking BDL group. A significant downregulation of mRNA expression has been detected for ACTA2 (0.43-fold), CTGF (0.36-fold), PDGFB (0.84-fold) and TNF- $\alpha$ (0.7-fold). Of note, in comparison to btgUGT1A-WT mice, coffee pre- and co-treatment showed less of a reduction of expression levels on fibrosis marker gene in the presence of UGT1A SNPs. Although coffee intake also resulted in a significant downregulation in btgUGT1A-SNP mice, higher mRNA expression levels for
ACTA2 (2.2-fold), CTGF (2.3-fold), TNF- $\alpha$ (1.2-fold) and CCL2 (1.6-fold) compared to htgUGT1A-WT mice were measured. These data indicate a less pronounced protective effect of coffee in carriers of the UGT1A SNP haplotype. In combination, these data suggest that reduced $U G T 1 A$ expression significantly attenuates the hepatoprotective effects of coffee on the expression of diverse biomarkers in the development of hepatic fibrosis.

\section{Reduced hepatic UGT1A expression during cholestatic liver fibrosis in coffee drinking btgUGT1A-SNP mice}

In order to investigate whether the observed hepatoprotective effect of coffee during biliary obstruction is based on differences in hepatic UGT1A expression, transcriptional $U G T 1 A$ regulation in $b \operatorname{tg} U G T 1 A$-SNP mice was quantified (Figure 7). Except for the isoforms UGT1A7 and UGT1A9, coffee consumption resulted in a significant transcriptional activation of $U G T 1 A$ genes in sham operated btgUGT1A-SNP mice, although the detected upregulation 
was less prominent as those obtained from equally treated btgUGT1A-WT mice. In contrast to the results observed in btgUGT1A-WT mice, UGT1A induction was reduced (UGT1A6 and UGT1A9) or absent in water drinking BDL mice carrying the UGT1A SNP haplotype. Even though a synergistic induction was detected after coffee $+\mathrm{BDL}$ co-treatment in btgUGT1A-SNP mice as well, absolute expression levels remained far below those observed in WT mice. In summary, btgUGT1A-SNP mice showed lower expression as well as a reduced responsiveness towards coffee during the development of cholestasis-induced liver fibrosis. As a consequence this may explain the reduced antioxidative and less protective effect of coffee during fibrogenesis observed in the presence of UGT1A SNPs.

\section{Discussion}

The beneficial effects of coffee in chronic liver diseases have been reported in numerous human and animal studies (46-49). Coffee is a potent inducer of the Nrf2 and AhR signalling pathways. Both are required for transcriptional UGT1A activation via xenobiotic response elements (XRE/ $\mathrm{AhR}$ ) and antioxidative response elements (ARE/Nrf2) binding motifs $(50,51)$. The coordinated regulation between both transcription factors and $U G T 1 A$ transcription links glucuronidation to xenobiotic-induced cellular protection and thus to the defence against oxidative stress (52). Oxidative stress represents a critical effector for the initiation of hepatocyte damage and hepatic fibrosis during cholestasis.

The present study investigated the influence of coffee on cytoprotective $U G T 1 A$ regulation in the situation of severe cholestasis induced by BDL. We demonstrate the significant activation of UGT1A1, UGT1A6, UGT1A7 and UGT1A9 mRNA expression in $b \operatorname{tg} U G T 1 A-W T$ mice as a result of BDL. These findings were expanded by studying the effects of coffee exposure. Coffee was found to further increase human $U G T 1 A$ gene expression in BDL animals resulting in a significant decrease of total serum bilirubin levels and a considerable reduction of aminotransferase activities. These findings provide evidence for hepatic protection linked to the activation of the UGT1A genes by coffee. With the intention of examining the opposite effect and expanding this analysis a $b \operatorname{tg} U G T 1 A-\mathrm{SNP}$ mouse line with genetically reduced $U G T 1 A$ expression because of the presence of 10 common UGT1A SNPs was used. In agreement with our hypothesis, expression levels were found to be lower and induction by coffee did not reach the levels observed with
btgUGT1A-WT mice. Moreover, the direct comparison between btgUGT1A-WT and btgUGT1A-SNP BDL animals confirmed a lower rate of fibrosis in WT compared to SNP mice suggesting a protective role of $U G T 1 A$ gene products for hepatic fibrogenesis in this situation. Because the retention of cytotoxic bile acids leads to severe cellular and tissue damage (53), the degree of activation of bile acid detoxifying mechanisms, including UGT1A-mediated conjugation with glucuronic acid, may be an important factor capable of influencing disease progression, and potential therapeutic interventions. Although the incidence of this variant SNP haplotype in other human populations than Caucasians is unknown, many of these polymorphisms were also found to exist at high frequencies in other ethnicities (Table 1). Therefore, an impaired UGT1Amediated cytoprotection during severe cholestasis may also constitute a relevant risk factor for humans with another ethnic background.

In order to show the contribution of UGT1A enzymes in hepatoprotection by exposure to coffee, fibrosis development, collagen deposition and mRNA expression of profibrotic factors were studied in both btgUGT1A mouse lines. HtgUGT1A-SNP mice showed significantly more ECM deposition after BDL and coffee + BDL co-treatment compared to mice containing the human wild type $U G T 1 A$ genotype. These results were accompanied by a comparable expression pattern of Col1a1 transcription, thereby confirming the results of the Sirius red staining fibrosis analysis. In line with these findings, the transcriptional induction of key genes related to fibrosis (CTGF, PDGFRB, CCL2 and TNF- $\alpha$ ) was higher in the presence of UGT1A SNPs compared to btgUGT1A-WT mice, which showed a higher degree of protection. Since the only difference between both animal models is the expression level of the UGT1A genes, these data suggest that UGT1As and their transcriptional activation play a protective role for fibrogenesis in cholestasis. In addition, an attenuated protective effect of coffee in BDL mice carrying the UGT1A SNP haplotype further corroborates that the coffee-mediated protection against hepatic fibrosis is to a substantial extent attributable to the ability of inducing $U G T 1 A$ gene products exerting antioxidative activity.

Recently published data also suggest that antioxidative properties of coffee leading to reduced oxidative stress in bile duct ligated Wistar rats can attenuate hepatic fibrosis (41). By sensing lipid peroxidation with 4-HNE antibody we demonstrated that $U G T 1 A$ function and inducibility significantly affects the antioxidative protective effect of 
coffee during cholestasis resulting in a lower amount of ROS-caused alternations of macromolecules and oxidative injury. As a consequence, the mechanism of action behind the coffee-mediated hepatoprotective effects is closely related to the coffee-induced enhancement of the antioxidative defence system in which UGT1As constitute a major player. As a result, reduced cellular protection against oxidative stress in $b \operatorname{tg} U G T 1 A$-SNP mice is likely to represent a crucial risk factor for enhanced fibrosis initiation and development.

In conclusion, we demonstrated that coffee exposure leads to protection against cholestasis-initiated liver fibrosis during BDL, which involves the regulation of UGT1A genes. The significant activation of human UGT1As is likely also associated with coffee exposure-mediated protective properties in other chronic liver diseases. Since coffee is a complex mixture containing a broad array of different chemical compounds (54), it would be of significant importance to identify the specific substances accountable for the detected $U G T 1 A$ upregulation. A detailed examination of the UGT1A enzymes paired with the identification of the relevant key constituents in coffee responsible for UGT1A activation could provide substantial benefits for risk evaluation of low-activity variants and for the assessment of UGTs as potential new therapeutic target and additional option to support the treatment of patients with cholestasis-related liver diseases.

\section{Acknowledgments}

Funding: This work was supported by grants from the Deutsche Forschungsgemeinschaft (DFG) Grant number STR 493/8-1 (to CPS).

\section{Footnote}

Conflicts of Interest: All authors have completed the ICMJE uniform disclosure form (available at https://hbsn. amegroups.com/article/view/10.21037/hbsn-20-9/coif). All authors have no conflicts of interest to declare.

Ethical statement: The authors are accountable for all aspects of the work in ensuring that questions related to the accuracy or integrity of any part of the work are appropriately investigated and resolved. All animal experiments were performed in accordance to the "German Animal-Protection Law" and approved by the North RhineWestphalian state-agency for Nature, Environment and
Consumer Protection (LANUV, Germany) under the file reference LANUV 84-02.04.2016.A483.

Open Access Statement: This is an Open Access article distributed in accordance with the Creative Commons Attribution-NonCommercial-NoDerivs 4.0 International License (CC BY-NC-ND 4.0), which permits the noncommercial replication and distribution of the article with the strict proviso that no changes or edits are made and the original work is properly cited (including links to both the formal publication through the relevant DOI and the license). See: https://creativecommons.org/licenses/by-nc-nd/4.0/.

\section{References}

1. Larsson SC. Coffee, tea, and cocoa and risk of stroke. Stroke 2014;45:309-14.

2. Loftfield E, Freedman ND, Dodd KW, et al. Coffee Drinking Is Widespread in the United States, but Usual Intake Varies by Key Demographic and Lifestyle Factors. J Nutr 2016;146:1762-8.

3. Heath RD, Brahmbhatt M, Tahan AC, et al. Coffee: The magical bean for liver diseases. World J Hepatol 2017;9:689-96.

4. Morisco F, Lembo V, Mazzone G, et al. Coffee and liver health. J Clin Gastroenterol 2014;48 Suppl 1:S87-90.

5. Marventano S, Salomone F, Godos J, et al. Coffee and tea consumption in relation with non-alcoholic fatty liver and metabolic syndrome: A systematic review and meta-analysis of observational studies. Clin Nutr 2016;35:1269-81.

6. Friedrich K, Smit M, Wannhoff A, et al. Coffee consumption protects against progression in liver cirrhosis and increases long-term survival after liver transplantation. J Gastroenterol Hepatol 2016;31:1470-5.

7. Kalthoff S, Ehmer U, Freiberg N, et al. Coffee induces expression of glucuronosyltransferases via the aryl hydrocarbon receptor and Nrf2 in liver and stomach. Gastroenterology 2010;139:1699-710, 1710.e1-2.

8. Strassburg CP, Kalthoff S. UDP-Glucuronosyltransferases. In: Anzenbacher P, Zanger UM. editors. Metabolism of Drugs and Other Xenobiotics. Wiley-VCH, 2012:67-116.

9. Dutton GJ. Glucuronidation of drugs and other compounds. Boca Raton, FL: CRC Boca Raton Press, 1980 .

10. Tukey RH, Strassburg CP. Human UDPglucuronosyltransferases: metabolism, expression, and disease. Annu Rev Pharmacol Toxicol 2000;40:581-616. 
11. Trottier J, Verreault M, Grepper S, et al. Human UDPglucuronosyltransferase (UGT)1A3 enzyme conjugates chenodeoxycholic acid in the liver. Hepatology 2006;44:1158-70.

12. Lankisch TO, Gillman TC, Erichsen TJ, et al. Aryl hydrocarbon receptor (AhR)-mediated regulation of the human estrogen and bile acid UDPGlucuronosyltransferase 1A3 gene. Arch Toxicol 2008;82:573-82.

13. Buckley DB, Klaassen CD. Induction of mouse UDPglucuronosyltransferase mRNA expression in liver and intestine by activators of aryl-hydrocarbon receptor, constitutive androstane receptor, pregnane $\mathrm{X}$ receptor, peroxisome proliferator-activated receptor alpha, and nuclear factor erythroid 2-related factor 2. Drug Metab Dispos 2009;37:847-56.

14. Erichsen TJ, Aehlen A, Ehmer U, et al. Regulation of the human bile acid UDP-glucuronosyltransferase 1A3 by the farnesoid $\mathrm{X}$ receptor and bile acids. J Hepatol 2010;52:570-8.

15. Strassburg CP, Nguyen N, Manns MP, et al. Polymorphic expression of the UDP-glucuronosyltransferase UGT1A gene locus in human gastric epithelium. Mol Pharmacol 1998;54:647-54.

16. Mackenzie PI, Hu DG, Gardner-Stephen DA. The regulation of UDP-glucuronosyltransferase genes by tissue-specific and ligand-activated transcription factors. Drug Metab Rev 2010;42:99-109.

17. Strassburg CP. Gilbert-Meulengracht's syndrome and pharmacogenetics: is jaundice just the tip of the iceberg? Drug Metab Rev 2010;42:168-81.

18. Wang Y, Kato N, Hoshida Y, et al. UDPglucuronosyltransferase $1 \mathrm{~A} 7$ genetic polymorphisms are associated with hepatocellular carcinoma in japanese patients with hepatitis $\mathrm{C}$ virus infection. Clin Cancer Res 2004;10:2441-6.

19. Tang KS, Lee CM, Teng HC, et al. UDPglucuronosyltransferase 1A7 polymorphisms are associated with liver cirrhosis. Biochem Biophys Res Commun 2008;366:643-8.

20. Bataller R, Brenner DA. Liver fibrosis. J Clin Invest 2005;115:209-18.

21. Liedtke C, Luedde T, Sauerbruch T, et al. Experimental liver fibrosis research: update on animal models, legal issues and translational aspects. Fibrogenesis Tissue Repair 2013;6:19.

22. Mormone E, George J, Nieto N. Molecular pathogenesis of hepatic fibrosis and current therapeutic approaches.
Chem Biol Interact 2011;193:225-31.

23. Hernandez-Gea V, Friedman SL. Pathogenesis of liver fibrosis. Annu Rev Pathol 2011;6:425-56.

24. Aktas C, Kanter M, Erboga M, et al. Melatonin attenuates oxidative stress, liver damage and hepatocyte apoptosis after bile-duct ligation in rats. Toxicol Ind Health 2014;30:835-44.

25. Kalthoff S, Landerer S, Reich J, et al. Protective effects of coffee against oxidative stress induced by the tobacco carcinogen benzo[alpha]pyrene. Free Radic Biol Med 2017;108:66-76.

26. Ehmer U, Kalthoff S, Fakundiny B, et al. Gilbert syndrome redefined: a complex genetic haplotype influences the regulation of glucuronidation. Hepatology 2012;55:1912-21.

27. Bosma PJ, Chowdhury JR, Bakker C, et al. The genetic basis of the reduced expression of bilirubin UDPglucuronosyltransferase 1 in Gilbert's syndrome. N Engl J Med 1995;333:1171-5.

28. Guillemette C, Ritter JK, Auyeung DJ, et al. Structural heterogeneity at the UDP-glucuronosyltransferase 1 locus: functional consequences of three novel missense mutations in the human UGT1A7 gene. Pharmacogenetics 2000;10:629-44.

29. Ehmer U, Lankisch TO, Meyer-Heithuis C, et al. Characterization and association of functional polymorphisms of the human hepatic UDPGlucuronosyltransferase UGT1A3. Hepatology 2004;40:277A.

30. Caillier B, Lepine J, Tojcic J, et al. A pharmacogenomics study of the human estrogen glucuronosyltransferase UGT1A3. Pharmacogenet Genomics 2007;17:481-95.

31. Iwai M, Maruo Y, Ito M, et al. Six novel UDPglucuronosyltransferase (UGT1A3) polymorphisms with varying activity. J Hum Genet 2004;49:123-8.

32. Saeki M, Saito Y, Jinno H, et al. Genetic polymorphisms of UGT1A6 in a Japanese population. Drug Metab Pharmacokinet 2005;20:85-90.

33. Lankisch TO, Vogel A, Eilermann S, et al. Identification and characterization of a functional TATA box polymorphism of the UDP glucuronosyltransferase 1A7 gene. Mol Pharmacol 2005;67:1732-9.

34. Strassburg CP, Vogel A, Kneip S, et al. Polymorphisms of the UDP-glucuronosyltransferase (UGT) $1 \mathrm{~A} 7$ gene in colorectal cancer. Gut 2002;50:851-6.

35. Tag CG, Sauer-Lehnen S, Weiskirchen S, et al. Bile duct ligation in mice: induction of inflammatory liver injury and fibrosis by obstructive cholestasis. J Vis Exp 
2015;(96):52438.

36. Liou GY, Storz P. Detecting reactive oxygen species by immunohistochemistry. Methods Mol Biol 2015;1292:97-104.

37. Muriel P, Arauz J. Coffee and liver diseases. Fitoterapia 2010;81:297-305.

38. Sánchez-Valle V, Chavez-Tapia NC, Uribe M, et al. Role of oxidative stress and molecular changes in liver fibrosis: a review. Curr Med Chem 2012;19:4850-60.

39. Laurent A, Perdu-Durand E, Alary J, et al. Metabolism of 4-hydroxynonenal, a cytotoxic product of lipid peroxidation, in rat precision-cut liver slices. Toxicol Lett 2000;114:203-14.

40. Gallelli CA, Calcagnini S, Romano A, et al. Modulation of the Oxidative Stress and Lipid Peroxidation by Endocannabinoids and Their Lipid Analogues. Antioxidants (Basel) 2018;7:93.

41. Arauz J, Zarco N, Hernandez-Aquino E, et al. Coffee consumption prevents fibrosis in a rat model that mimics secondary biliary cirrhosis in humans. Nutr Res 2017;40:65-74.

42. Ruhl CE, Everhart JE. Coffee and tea consumption are associated with a lower incidence of chronic liver disease in the United States. Gastroenterology 2005;129:1928-36.

43. Setiawan VW, Wilkens LR, Lu SC, et al. Association of coffee intake with reduced incidence of liver cancer and death from chronic liver disease in the US multiethnic cohort. Gastroenterology 2015;148:118-25; quiz e15.

44. Costentin CE, Roudot-Thoraval F, Zafrani ES, et al. Association of caffeine intake and histological features of chronic hepatitis C. J Hepatol 2011;54:1123-9.

45. Kennedy OJ, Roderick P, Buchanan R, et al. Systematic review with meta-analysis: coffee consumption and the risk of cirrhosis. Aliment Pharmacol Ther 2016;43:562-74.

46. Klatsky AL, Morton C, Udaltsova N, et al. Coffee, cirrhosis, and transaminase enzymes. Arch Intern Med 2006;166:1190-5.

47. Modi AA, Feld JJ, Park Y, et al. Increased caffeine consumption is associated with reduced hepatic fibrosis. Hepatology 2010;51:201-9.

48. Saab S, Mallam D, Cox GA, 2nd, et al. Impact of coffee on liver diseases: a systematic review. Liver Int 2014;34:495-504.

49. Salomone F, Galvano F, Li Volti G. Molecular Bases Underlying the Hepatoprotective Effects of Coffee. Nutrients 2017;9:85.

50. Kalthoff S, Ehmer U, Freiberg N, et al. Interaction between oxidative stress sensor Nrf2 and xenobioticactivated aryl hydrocarbon receptor in the regulation of the human phase II detoxifying UDP-glucuronosyltransferase 1A10. J Biol Chem 2010;285:5993-6002.

51. Ishikawa T, Takahashi S, Morita K, et al. Induction of AhR-mediated gene transcription by coffee. PLoS One 2014;9:e102152.

52. Lu Y, Zhuge J, Wang X, et al. Cytochrome P450 2E1 contributes to ethanol-induced fatty liver in mice. Hepatology 2008;47:1483-94.

53. Perreault M, Bialek A, Trottier J, et al. Role of glucuronidation for hepatic detoxification and urinary elimination of toxic bile acids during biliary obstruction. PLoS One 2013;8:e80994.

54. Higdon JV, Frei B. Coffee and health: a review of recent human research. Crit Rev Food Sci Nutr 2006;46:101-23.
Cite this article as: Landerer S, Kalthoff S, Strassburg CP. UDP-glucuronosyltransferases mediate coffee-associated reduction of liver fibrosis in bile duct ligated humanized transgenic UGT1A mice. HepatoBiliary Surg Nutr 2021;10(6):766-781. doi: 10.21037/hbsn-20-9 